\title{
Direct comparison of two commercially available computer programs for analysing DNA fingerprinting gels
}

\author{
R. J. SEWARD, B. EHRENSTEIN*, H. J. GRUNDMANN* and K. J. TOWNER \\ Department of Microbiology and PHLS Laboratory, University Hospital, Queen's Medical Centre, Nottingham \\ NG7 $2 \mathrm{UH}$ and *Institut für Umweltmedizin und Krankenhaushygiene, Klinikum der Albert-Ludwigs-Universität, \\ 79106 Freiburg, Germany
}

\begin{abstract}
Randomly amplified polymorphic DNA (RAPD) fingerprints were generated with M13 and DAF4 primers for 25 isolates of Acinetobacter spp. obtained from 16 different hospitals situated in 12 countries. The overall robustness of the algorithms and the reproducibility of the cluster analysis results generated by two commercially available computer programs (GelCompar and DENDRON) for analysing DNA fingerprinting gels were tested by examining the same set of fingerprinting data independently in two laboratories with the different software packages. Both programs were efficient at recognising and grouping strains with closely similar RAPD fingerprints, i.e., strains which might be expected to have a close epidemiological or evolutionary relationship. However, the relationships suggested for less closely related strains showed considerable variation in terms of the overall similarity or percentage correlation values suggested by the programs. It was concluded that both programs were useful tools for indicating close genotypic relationships between individual strains, but that epidemiological conclusions based on the similarity or correlation values (or the dendrograms derived from them) obtained for less closely related strains should be treated with considerable caution.
\end{abstract}

\section{Introduction}

Rapid DNA fingerprinting methods [1-6] are being used increasingly in many microbiology laboratories for epidemiological typing of an ever-increasing range of bacteria. In almost all cases, these methods involve comparison of DNA band patterns that are visualised following electrophoresis and which are then assumed to reflect genetic relatedness. Banding patterns obtained for a small number of strains on a single electrophoresis gel can usually be compared visually, but difficulties arise when attempting to compare visually the fingerprint banding patterns obtained from a large number of isolates examined on different gels over extended time intervals or in different laboratories. Various computer-assisted systems have been developed in an attempt to deal with these difficulties, to create databases for continuing or retrospective epidemiological studies and to demonstrate clustering for groups of genetically similar strains. Although a more

Received 4 Sept. 1996; accepted 16 Sept. 1996.

Corresponding author: Dr K. J. Towner. advanced approach involving on-line laser fluorescence analysis of DNA fragments separated on DNA sequencing gels has been described [7,8], most of these systems involve scanning photographs or autoradiographs of banding patterns to digitise a gel image and then incorporate the banding patterns into a database. The digitised image can then be processed and used to generate a matrix of similarity coefficients between all possible pairs of strains, leading in turn to a dendrogram in which all patterns are placed in a horizontal 'tree' with branching dictated by similarity coefficients. New strains can be compared with strains analysed previously provided that the same normalisation standards are run on each individual gel, and any subset of strains in a study can be compared in a dendrogram or mixed with any other subset in a dendrogram.

Several different programs for computers with either Macintosh or Windows-based operating systems are now available commercially for analysing DNA fingerprinting gels. Although largely automated, these programs still require a limited amount of operator intervention to deal with artifacts recognised by the automated system. Such programs are being used 
increasingly by clinical microbiology laboratories and many published studies now attach increasing significance to dendrograms illustrating the results of epidemiological investigations - but studies comparing the results generated by these semi-automated systems have yet to be performed. The work described in this paper was designed to test the overall robustness of the algorithms used in the software of two of these programs - as well as the reproducibility of the cluster analysis results generated - by examining the same set of digitised fingerprinting gel images independently in two different laboratories.

\section{Materials and methods}

\section{Bacteria}

For the comparative purposes of this study, DNA fingerprints were generated for 25 isolates of Acinetobacter spp. obtained from 16 different hospitals in 12 countries. The 25 strains examined (Table 1) had all been identified originally as $A$. baumannii on the basis of biochemical tests performed on initial isolation at the various source hospitals. However, subsequent analysis by the tDNA profile method of Ehrenstein et al. [9] demonstrated that three of the strains (indicated in Table 1) belonged to Acinetobacter genospecies 3 . The strains were stored at $-70^{\circ} \mathrm{C}$ in Skim Milk Powder (Oxoid) $10 \% \mathrm{w} / \mathrm{v}$ in sterile distilled water and then subcultured on to CLED Agar (Oxoid) and incubated overnight at $37^{\circ} \mathrm{C}$ immediately before extraction of genomic DNA.

Table 1. Isolates of Acinetobacter spp. used to generate the RAPD fingerprints for the study

\begin{tabular}{lll}
\hline Strain & Source & Identification* \\
\hline CW1 & Nottingham, England & A. baumannii \\
CW5 & Nottingham, England & A. baumannii \\
CW11 & Bristol, England & Acinetobacter sp. 3 \\
CW12 & Bristol, England & Acinetobacter sp. 3 \\
CW13 & Rotterdam, The Netherlands & A. baumannii \\
CW14 & Venlo, The Netherlands & A. baumannii \\
CW20 & Paris, France & A. baumannii \\
CW22 & Paris, France & A. baumannii \\
CW27 & Cardiff, Wales & A. baumannii \\
CW28 & East Glamorgan, Wales & A. baumannii \\
CW31 & Perth, Australia & Acinetobacter sp. 3 \\
CW32 & Perth, Australia & A. baumannii \\
G1 & Cape Town, South Africa & A. baumannii \\
G5 & Cape Town, South Africa & A. baumannii \\
S2 & Singapore & A. baumannii \\
S4 & Singapore & A. baumannii \\
A1 & Buenos Aires, Argentina & A. baumannii \\
A16 & Buenos Aires, Argentina & A. baumannii \\
B1 & Berlin, Germany & A. baumannii \\
B5 & Berlin, Germany & A. baumannii \\
D1 & Freiburg, Germany & A. baumannii \\
D2 & Freiburg, Germany & A. baumannii \\
ESP41 & Barcelona, Spain & A. baumannii \\
ESP77 & Barcelona, Spain & A. baumannii \\
I133 & Trieste, Italy & A. baumannii \\
\hline
\end{tabular}

* Strain identification was by the tDNA fingerprinting method of Ehrenstein et al. [9].

\section{Release of genomic DNA and generation of DNA fingerprints}

Total genomic DNA was released from bacteria by resuspending 3-4 small colonies in a $0.5-\mathrm{ml}$ microfuge tube containing $100 \mu \mathrm{l}$ of sterile distilled water and heating at $95^{\circ} \mathrm{C}$ for $15 \mathrm{~min}$. The resulting crude DNA suspensions were cooled on ice, centrifuged for $30 \mathrm{~s}$ at $12000 \mathrm{~g}$ and stored at $-20^{\circ} \mathrm{C}$ until required. Randomly amplified polymorphic DNA (RAPD) fingerprints were generated with either: (i) M13 core region primer (5'GAGGGTGGCGGTTCT-3'), used with the amplification conditions described by Grundmann et al. [7]; or (ii) DAF4 primer $\left(5^{\prime}\right.$-CGGCAGCGCC-3'), used with the amplification conditions described by WiedmannAl-Ahmad et al. [10]. The primers used were customsynthesised by Oswel DNA Service (Southampton). Amplification products were photographed (Polaroid Type 667 Film) after separation on agarose $2 \% \mathrm{w} / \mathrm{v}$ gels, staining with ethidium bromide and visualisation on a UV-transilluminator. Molecular size markers (pGEM DNA Markers; Promega) were loaded at least once every seven lanes on gels to allow inter-gel normalisation and comparison.

\section{Production of digitised gel images}

Photographs of RAPD fingerprints from different gels were scanned with a UMAX UC840 Scanner (Leicester Computer Centre) equipped with Adobe Photoshop Macintosh v.2.5.1 software and were then used to form a composite image for the 25 strains with each primer. Digitised images were saved to diskettes in the PICT and TIFF formats. The diskettes containing the digitised data were readily exchangeable between the two laboratories participating in the study so that identical gel images, and the corresponding digitised data, were then used for further analysis by the two programs included in the study.

\section{Analysis of digitised gel images}

The two programs used to analyse the digitised gel data were DENDRON v.2.0 (Solltech Inc., Oakdale, IA, USA) for computers running the Macintosh operating system and GelCompar v.3.10 (Applied Maths, Kortrijk, Belgium) for computers running the Windows operating system. Both programs normalise data from separate electrophoresis gels according to either internal size standards added to each track or (as in this study) sets of molecular size standards run at regular intervals in separate tracks. Alignment by the computer of the size standards allows inter- and intra-gel inconsistencies and variations in electrophoresis conditions to be corrected.

The DENDRON program identifies the positions and intensities of the bands in each lane of a gel and then calculates a similarity coefficient $\left(\mathrm{S}_{\mathrm{AB}}\right)$ for every pair of strains. For the purposes of this study, the $\mathrm{S}_{\mathrm{AB}}$ values were computed solely on the basis of band 
positions by the Dice coefficient [11]. The $\mathrm{S}_{\mathrm{AB}}$ values are presented in a matrix and then used to generate a dendrogram by the unweighted pair group method using arithmetic averages (UPGMA) in which the two or more strains with the highest $\mathrm{S}_{\mathrm{AB}}$ value are grouped into a unit with a connection (or branch point) corresponding to that $\mathrm{S}_{\mathrm{AB}}$ value along the horizontal axis of the dendrogram. The process continues in the direction of lower $\mathrm{S}_{\mathrm{AB}}$ values until the dendrogram is complete.

The GelCompar program operates in a very similar fashion to DENDRON, but generates dendrograms, with results expressed in terms of percentage correlation values, based on similarity matrices calculated from the Pearson product-moment correlation coefficient [12]. This coefficient is a measure of the simple linear correlation between the overall density profile of two different fingerprint banding patterns. It is principally independent of the relative intensity of individual bands and can assume any value between 1.0 and -1.0 . Thereby, $1.0(100 \%)$ indicates a complete match and -1.0 indicates an inverse symmetry between two profiles. It is important to note that absolute values generated by Pearson's correlation coefficient cannot be compared directly with other coefficients, such as Dice indices, generated on the basis of banding locations.

\section{Results}

The RAPD fingerprints obtained with the M13 primer consisted of between one and 16 DNA bands, while those obtained with the DAF4 primer consisted of between one and 10 bands. Several different gels were required to analyse the fingerprints obtained for all 25 strains. Figs. 1 and 2 show the composite images

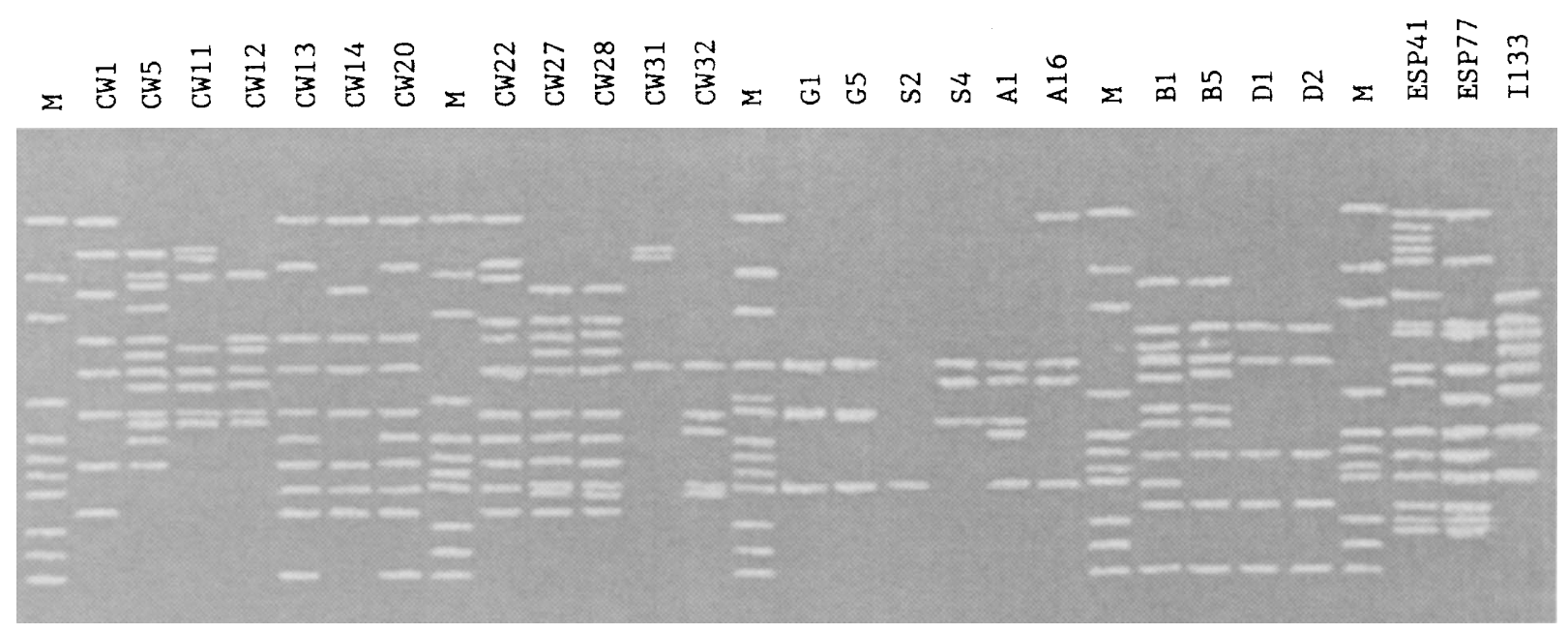

Fig. 1. Composite computer image obtained following scanning of the M13 fingerprinting gels. Strain designations are indicated (M, pGEM DNA markers).

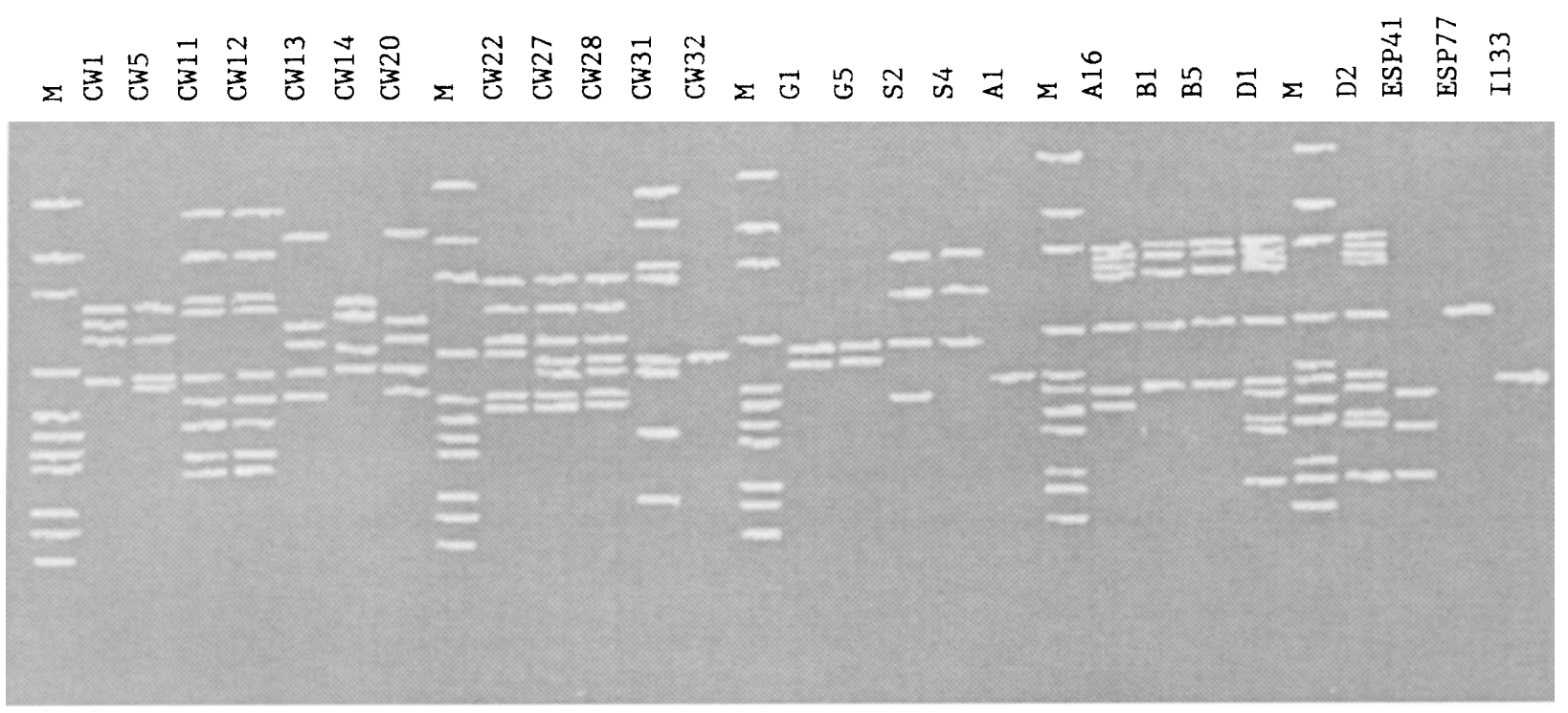

Fig. 2. Composite computer image obtained following scanning of the DAF4 fingerprinting gels. Strain designations are indicated ( $\mathrm{M}$, pGEM DNA markers). 
obtained following scanning of the M13 and DAF4 fingerprinting gels. These images were saved to diskette and then exchanged between the two participating laboratories for analysis by the DENDRON and GelCompar programs.

Figs. $3 \mathrm{a}$ and $\mathrm{b}$ show the dendrograms obtained from cluster analysis of the RAPD fingerprints obtained with the M13 primer by the DENDRON and GelCompar programs, respectively. The dendrograms identified the same close clustering relationships for groups of two or more related strains; however, the overall gross structures of the dendrograms showed decreasing reproducibility in line with decreasing similarity, although isolated 'unrelated' strains (e.g., strains CW31 and 1133) were not clustered with other strains by either program.

Similar results were obtained from cluster analysis of the RAPD fingerprints obtained with the DAF4 primer (Figs. $4 \mathrm{a}$ and $\mathrm{b}$ ). One major anomaly was observed in that strains ESP77 and CW32 were recognised as identical by the DENDRON program, but were unlinked by GelCompar. However, detailed examination of the band data and linkages for these strains revealed that strains ESP77 and CW32 yielded only one band each - both of similar size - following RAPD fingerprinting with the DAF4 primer (Fig. 2). These two bands were recognised as identical by the DENDRON program, but were unlinked by the GelCompar program. RAPD fingerprinting with the M13 primer yielded five and 12 DNA bands, respectively, for strains ESP77 and CW32 (Fig. 1) and both programs indicated that these two strains were genotypically unrelated (Figs. 3a and b). With the exception of these two strains, both programs indicated the same clustering relationships between groups of two or more closely related strains, but - as found with the RAPD fingerprints generated with the M13 primer - the gross structures of the dendrograms were somewhat variable and showed an increasing lack of reproducibility in line with decreasing similarities.

\section{Discussion}

Computer-assisted strategies are becoming increasingly important for assessing the genetic relatedness of micro-organisms in epidemiological studies, and are essential for analysis of complex DNA fingerprints and in cases where strains from different geographical locations are being compared over significant time periods. An increasing number of published studies now reach important epidemiological conclusions on the basis of cluster analysis results presented in the form of dendrograms. The work described in this paper was not concerned with discerning the epidemiological relationships of the bacterial isolates studied per se, but aimed to compare the strain groupings suggested by the two different programs included in the study when analysing the same sets of fingerprinting data. In this respect the study was able to demonstrate clearly some of the advantages and the limitations of the two systems used.

On the positive side, both programs were efficient at recognising and grouping strains with closely similar genotypic fingerprints, i.e., strains which might be expected to have a close epidemiological or evolutionary relationship. This is particularly useful in the case of complex fingerprints where minor band changes between strains are sometimes difficult to assess objectively by visual means. Some minor disagreements in $\mathrm{S}_{\mathrm{AB}}$ and percentage correlation values between closely related strains were observed, but it is not valid to compare these values directly because of the different calculation methods used by the two programs. The only major disagreement between the close relationships suggested by the programs involved two strains that produced only one 'similar' band each following RAPD fingerprinting with the DAF4 primer. These single bands were recognised as identical by one program, but as different by the other. This observation emphasises the continued need for manual assessment and interpretation of automated results - as well as the need to ensure that findings are based on sufficiently complex fingerprints - and to compare results obtained by more than one fingerprinting method.

On the negative side, the relationships suggested by the dendrograms for less closely related strains showed considerable variations in terms of the overall similarities suggested by the programs. This reflects, in part at least, the precise mechanism used by each program to calculate strain relationships. The correlation coefficients are first presented as a matrix and then used to generate a dendrogram by means of an UPGMA program in which the two or more strains with the highest correlation value are grouped into a unit with a connection or branch point corresponding to that value on the horizontal axis of the dendrogram. The process then continues in the direction of lower correlation values by searching for the strain-strain, strain-unit or unit-unit with the next highest value. Units consist of two or more strains, so that the branch point for strain-unit or unit-unit combinations is determined by the average correlation value between members of a unit and the strain or unit with which it is paired. For this reason, branch points involving a unit are not as accurate as the individually computed correlation values for two individual strains in the original correlation matrix.

Overall, it was concluded that - provided the original fingerprint patterns generated were of sufficient complexity to distinguish between unrelated strains both programs were efficient at clustering strains with a relatively high similarity coefficient. Similar close 


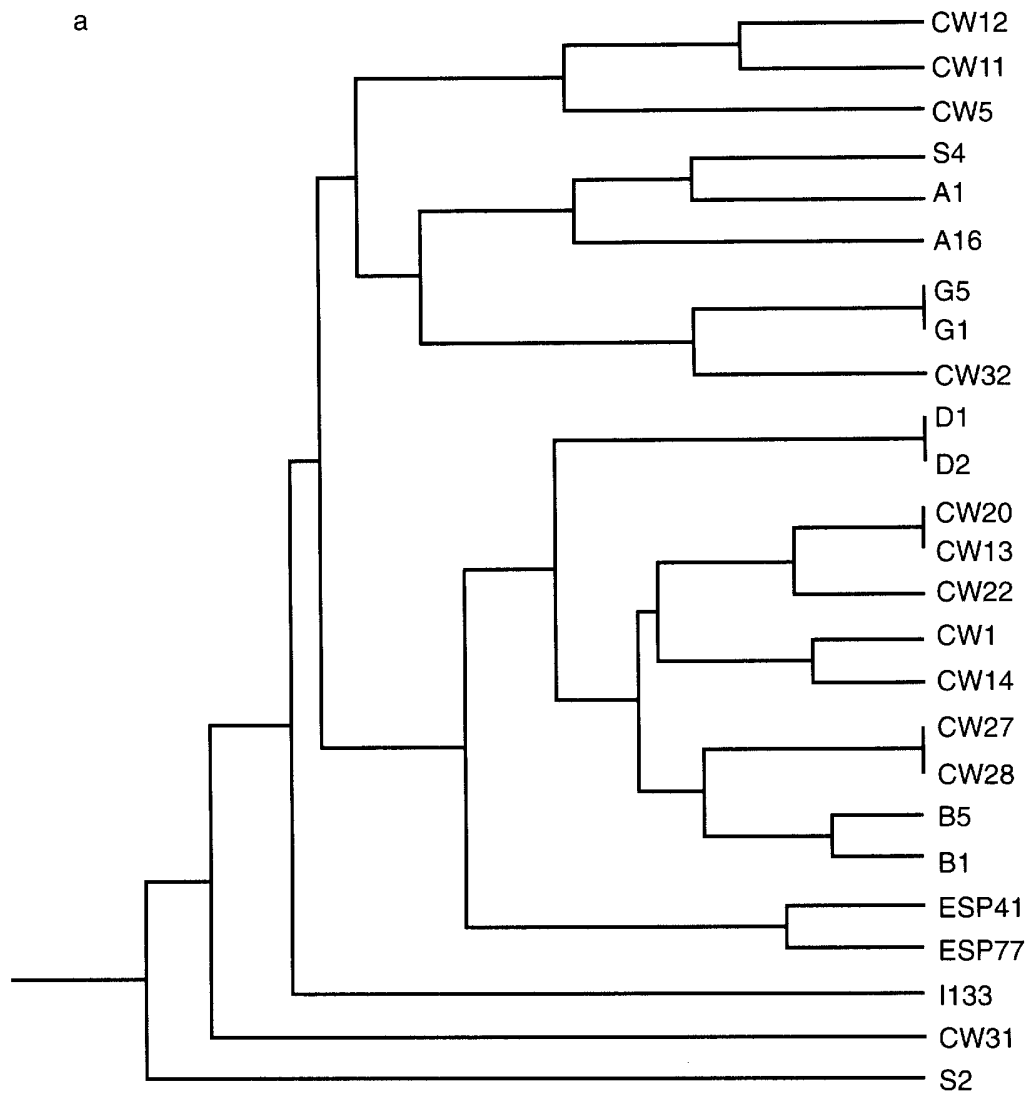

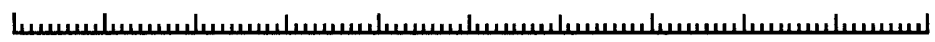

$\begin{array}{llllllllllll}0 & .1 & .2 & .3 & .4 & .5 & .6 & .7 & .8 & .9 & & 1\end{array}$

b

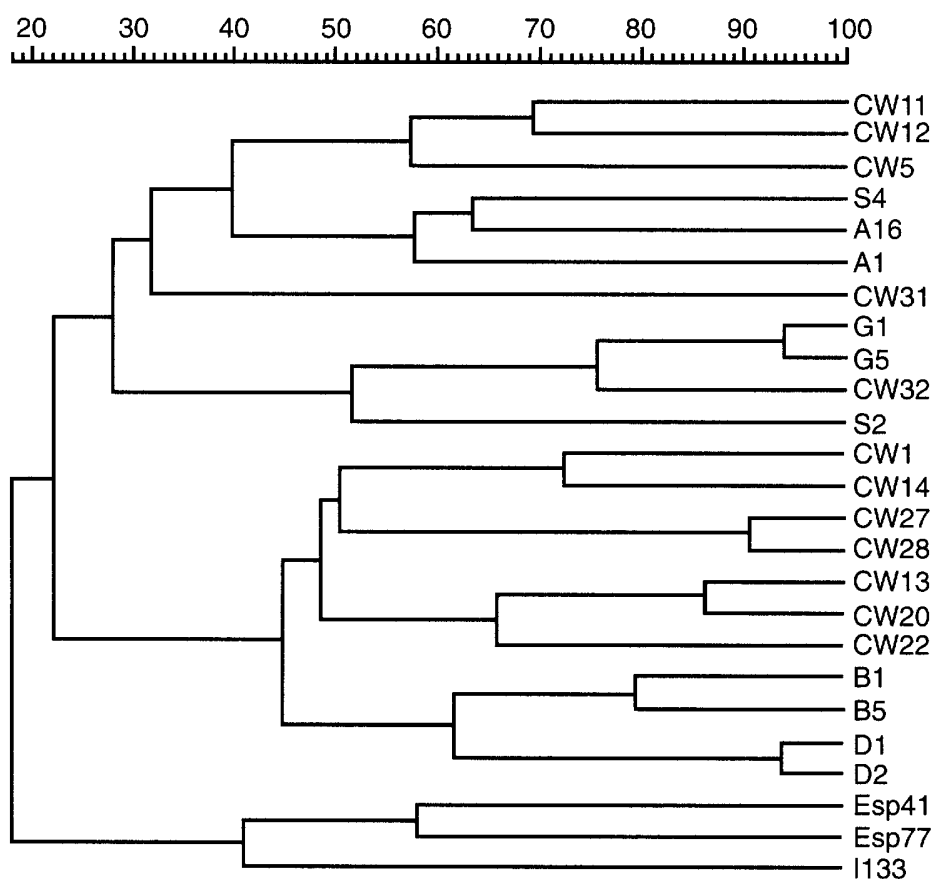

Fig. 3. Dendrograms obtained from analysis of the composite M13 fingerprinting image with (a) DENDRON and (b) GelCompar. 
a

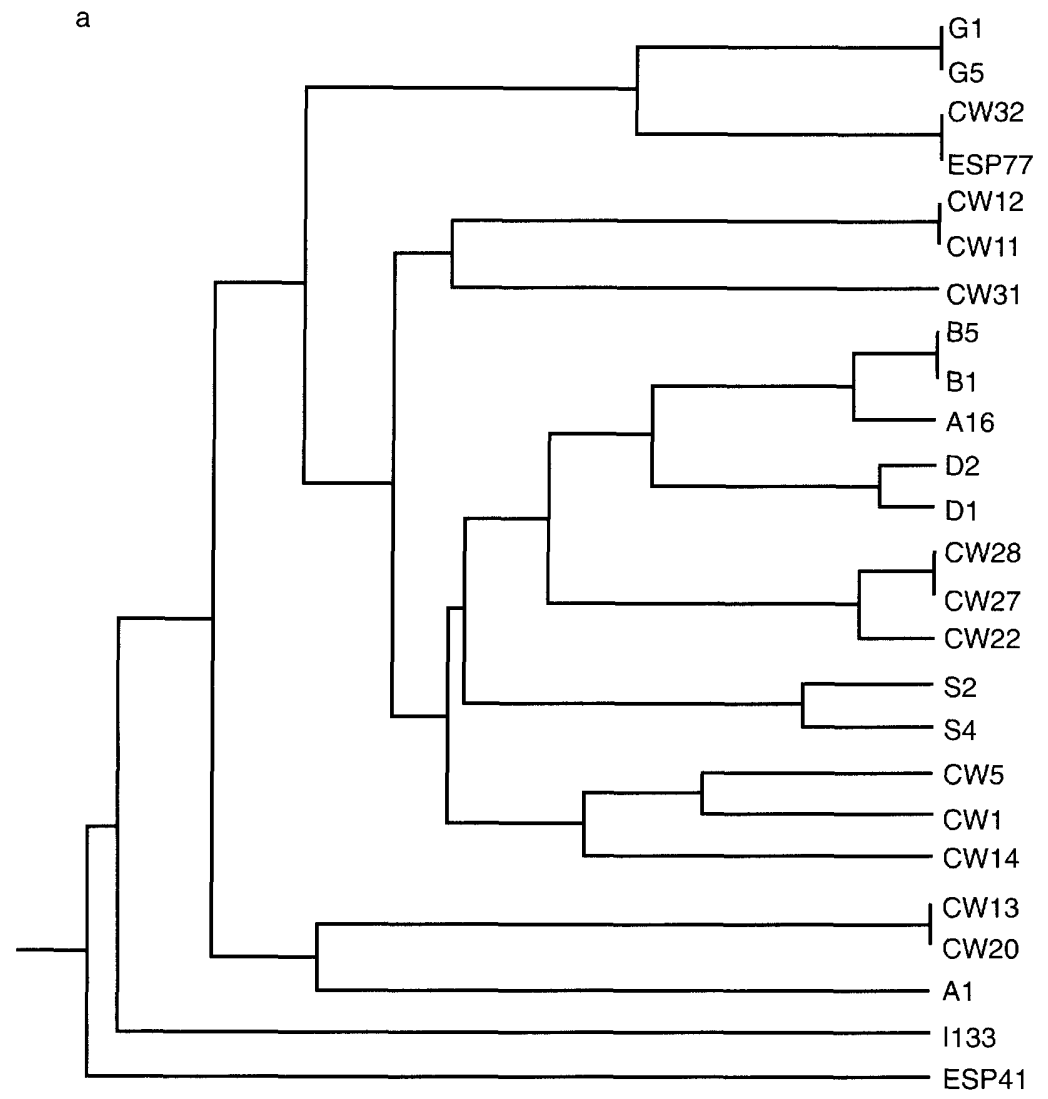

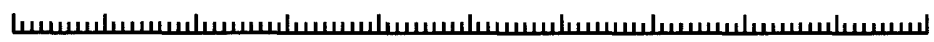

$\begin{array}{lllllllllllll}0 & .1 & .2 & .3 & .4 & .5 & .6 & .7 & .8 & .9 & 1 & \mathrm{~S} & \mathrm{AB}\end{array}$

b

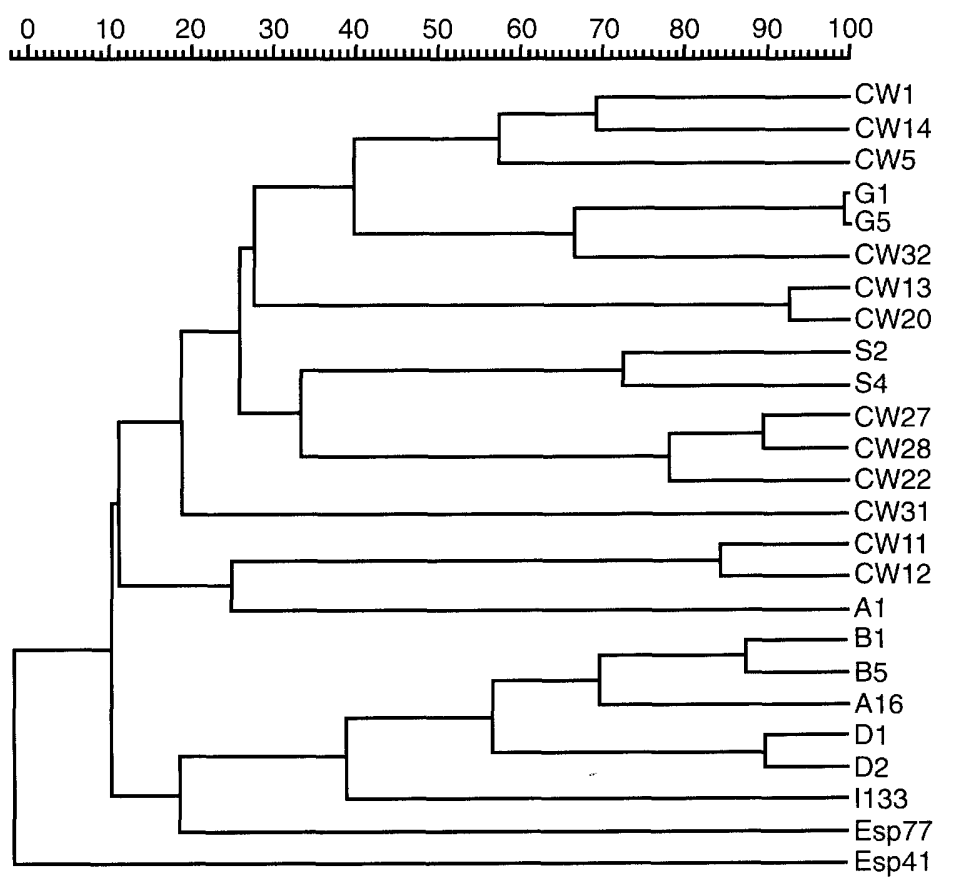

Fig. 4. Dendrograms obtained from analysis of the composite DAF4 fingerprinting image with (a) DENDRON and (b) GelCompar. 
clusters of strains were obtained with both programs. However, although the similarity coefficients used to generate such clusters are relatively straightforward computations, correlating these values with 'genetic distance' (i.e., the number of generations separating strains from a common clonal origin) is much more difficult (and may be impossible) and requires further experimentation. In particular, the results presented in this paper indicated that relatively low $\mathrm{S}_{\mathrm{AB}}$ or percentage correlation values showed wide variation between the two programs and that epidemiological conclusions based on such values, or the dendrograms generated from them, should be treated with considerable caution. Rigorous normalisation of sample lanes on a gel is mandatory to allow accurate intra- and inter-gel comparisons. Addition of molecular size markers to each and every lane would probably yield optimum results; alternatively, as indicated by the results of this study, a set of molecular size markers run in at least every fifth or sixth lane would seem to be the minimum requirement for acceptable normalisation of gels. Such size markers should span the entire range of DNA band sizes being analysed. Finally, while both of the programs examined in this study were shown to be useful tools for indicating genotypic relationships between individual strains and are probably essential for long-term epidemiological comparisons - it was concluded that assessment of the results and occasional intervention by a trained microbiologist is still essential to detect and correct artifacts introduced by the automated systems.

We are indebted to all those colleagues who donated the strains of Acinetobacter used to generate the RAPD fingerprints for this study. We gratefully acknowledge support for our collaborations from The British Council, the Deutscher Akademischer Austauschdienst (DAAD) and the Ciba Fellowship Trust.

\section{References}

1. Maslow JN, Mulligan ME, Arbeit RD. Molecular epidemiology: application of contemporary techniques to the typing of microorganisms. Clin Infect Dis 1993; 17: 153-164.

2. Mayer LW. Use of plasmid profiles in epidemiologic surveillance of disease outbreaks and in tracing the transmission of antibiotic resistance. Clin Microbiol Rev 1988; 1: 228-243.

3. Owen RJ. Chromosomal DNA fingerprinting - a new method of species and strain identification applicable to microbial pathogens. J Med Microbiol 1989; 30: 89-99.

4. Goering R. Molecular epidemiology of nosocomial infection: analysis of chromosomal restriction fragment patterns by pulsed-field gel electrophoresis. Infect Control Hosp Epidemiol 1993; 14: $595-600$.

5. Bingen $E$, Denamur $E$, Elion $J$ Use of ribotyping in epidemiological surveillance of nosocomial outbreaks. Clin Microbiol Rev 1994; 7: 311-327.

6. Van Belkum A. DNA fingerprinting of medically important microorganisms by use of PCR. Clin Microbiol Rev 1994; 7: 174-184.

7. Grundmann $\mathrm{H}$, Schneider $\mathrm{CH}$, Tichy HV et al. Automated laser fluorescence analysis of randomly amplified polymorphic DNA: a rapid method for investigating nosocomial transmission of Acinetobacter baumannii. J Med Microbiol 1995; 43: $446-451$.

8. Webster CA, Towner KJ, Humphreys H, Ehrenstein B, Hartung $D$, Grundmann $H$. Comparison of rapid automated laser fluorescence analysis of tDNA fingerprints with four other computer-assisted approaches for studying relationships between Acinetobacter baumannii isolates. $J$ Med Microbiol 1996; 44: $185-194$.

9. Ehrenstein B, Bernards AT, Dijkshoorn L et al. Acinetobacter species identification using tRNA fingerprinting. $J$ Clin Microbiol 1996; 34: 2414-2420.

10. Wiedmann-al-Ahmad M, Tichy HV, Schön G. Characterization of Acinetobacter type strains and isolates obtained from wastewater treatment plants by PCR fingerprinting. Appl Environ Microbiol 1994; 60: 4066-4071.

11. Struelens MJ and the Members of the European Study Group on Epidemiological Markers (ESGEM) of the European Society for Clinical Microbiology and Infectious Discases (ESCMID). Consensus guidelines for appropriate use and evaluation of microbial epidemiologic typing systems. Clin Microbiol Infect 1996; 2: 1-11.

12. Sneath PHA, Sokal RR. Numerical taxonomy, the principles and practice of numerical classification. San Francisco, WH Freeman. 1973. 